\title{
Application of the Electron Theory of Sintering to the Ferrite Systems
}

\author{
A.V. KopayeV And V.S. Bushkova \\ V. Stefanyk Precarpathian National University, Ivano-Frankivsk, Ukraine
}

\begin{abstract}
The compositions of magnesium-zinc ferrites $\left(\mathrm{Mg}_{0.56} \mathrm{Zn}_{0.44}\right)_{1-y} \mathrm{Fe}_{2+2 y / 3} \mathrm{O}_{4}$, where $y=-0.05,0,+0.05$ were sintered at temperatures 1230 and $1280^{\circ} \mathrm{C}$. The analysis of the kinetics of sintering was carried out by using the Ivensen model. It was shown that at decrease of $y$ the sintering rate increases. The analysis of the electronic configurations of the cations in the ferrite composition showed the possibility of applying the Samsonov electronic theory of sintering powders to them. Magnetic permeability of the studied chemical compositions increases with increase of the density of ferrites as well as with the temperature and the time of isothermal soaking.
\end{abstract}

PACS numbers: 31.10.+z, 36.20.Hb, 66.30.-h, 77.84.Bw, 81.20.Ev, 85.70.Ge

\section{Introduction}

Ferrites are often derived from metal oxides by the method of powders sintering at high temperatures and controlled gas atmosphere. Studies have shown that the electromagnetic properties of ferrites depend strongly on the temperature and oxygen regimes of sintering and the density of products $[1,2]$. The process of density increasing in the solid state at high temperature depends on several factors. Particularly, the atomic number of metal $\mathrm{M}$ in the formula of the ferrite $\mathrm{MFe}_{2} \mathrm{O}_{4}$ and nonstoichiometry of this compound can greatly change the course of ferrite sintering $[3,4]$. The mechanism of such dependence is not entirely clear yet.

There are several theoretical models that help to understand the mechanisms of solid state processes taking place during sintering. These theories describe the processes occurring in the size of atoms and groups of atoms. The electron theory of metal powders sintering has been developed by Samsonov [5, 6]. It allows to understand the influence of the electronic structure of elements on the processes of mass transfer. However, the application of this theory for the sintering of oxide materials is worth considering. In the present work we study the influence of nonstoichiometry on the kinetics of sintering of MgZn-ferrites and their permeability. At that we use the Samsonov theory for analysis of the influence of electronic configuration of cations on the processes of mass transfer at high temperatures.

\section{Theoretical basis of the research}

During sintering in oxide systems, moving of masses is carried out mainly through the diffusion of relatively heavy metal cations. As it is known, the temperature dependence of the diffusion coefficient is described by expression

$$
D=D_{0} \exp (-E / k T),
$$

where $E$ is the activation energy for diffusion and $D_{0}$ is the preexponential factor. This factor depends on the electronic structure of the diffusant and the distance between ions (atoms, molecules) in the material. As it is shown in the Samsonov configuration model of the substance structure, the ions (atoms) with a stable electronic configuration are characterized by high speed movement and high value of $D_{0}$. Particularly, the stable configurations are $s^{2} p^{6}, 3 d^{5}, 3 d^{10}[5]$.

In a number of ferrites of $\mathrm{M}_{n} \mathrm{M}_{m}^{\prime} \mathrm{Fe}_{2}^{3+} \mathrm{O}_{4}$ metal ions $\mathrm{M}$ and $\mathrm{M}^{\prime}$ can form more stable electronic configurations like $3 d^{10}\left(\mathrm{Zn}^{2+}\right), 2 p^{6}\left(\mathrm{Mg}^{2+}\right)$ than other ions such as $\mathrm{Ni}^{2+}$ $\left(3 d^{8}\right), \mathrm{Mn}^{3+}\left(3 d^{4}\right)$. State of electronic system excitation of ionic crystalline oxides and accordingly the actual electronic configuration of cations will depend on the nature of interaction with the surrounding ions. For example, during sintering of ferrites at temperatures above $1200^{\circ} \mathrm{C}$ iron ions $(3+)$ tend to reduce due to the reaction [7]:

$$
\mathrm{Fe}^{3+}+(1 / 2) \mathrm{O}^{2-} \leftrightarrow \mathrm{Fe}^{2+}+(1 / 4) \mathrm{O}_{2} \uparrow .
$$

Recharging of iron ions from $\mathrm{Fe}^{2+}\left(3 d^{6}\right)$ to $\mathrm{Fe}^{3+}\left(3 d^{5}\right)$ [8] reduces the stability of their electronic configuration, that should reduce their diffusion mobility in accordance with the Samsonov theory. To evaluate the diffusion mobility of other ions we used the published experimental data [9], particularly, the results of the study of diffusion of $\mathrm{M}^{2+}$ cations $\left(\mathrm{Fe}^{2+}, \mathrm{Zn}^{2+}, \mathrm{Mg}^{2+}\right.$, etc.) in the ferrites. Ferrites, according to the experimental procedure [9], were brought into contact with the surface of single crystal $\mathrm{MgO}$, and divalent cations diffuse from the ferrite in a single crystal. The concentration of cations in $\mathrm{MgO}$ decreases regularly depending on the distance to the surface of a single crystal. The concentration of iron in $\mathrm{MgO}$ decreased faster than the concentration of zinc. Consequently, the diffusion coefficient of zinc is higher 
than that of iron. This can be explained within the configuration model so that the electronic configuration of $\mathrm{Zn}$ is more stable in comparison with the one of iron in the above cited reasons. It was shown by tracers that the diffusion coefficient of iron ferrite is higher than that of nickel. It is obviously, caused by the less stable electronic configuration of the $\mathrm{Ni}^{2+}$ cation $\left(3 d^{8}\right)$.

Since the electronic configuration of $\mathrm{Mg}^{2+}$ ions is more stable than the configuration $\mathrm{Ni}^{2+}$, their diffusion mobility should be higher. This may explain the low porosity of stoichiometric magnesium ferrite in comparison with nickel ferrite sintered at the same conditions [10]. Thus, the Samsonov configuration model allows us to explain the difference of the diffusion mobilities of some cations.

Obviously, sintering of oxides is conditioned not only by the electron configuration of atoms, but also by partial structure imperfections [11]. One of the physically and mathematically well-developed theories of sintering is the Ivensen model $[12,13]$. It provides two main processes taking place simultaneously - reducing of macro-defects (like pores) and elimination of micro-defects (like atomic structure imperfections). The pores are filled with the substance by the law of viscous flow

$$
\frac{1}{v} \frac{\mathrm{d} v}{\mathrm{~d} \tau}=-\kappa N \exp \left(-\frac{E_{\kappa}}{R T}\right),
$$

where $v$ is the pore volume, $N$ is the concentration of imperfections, $E_{\kappa}$ is the activation energy of flow of the substance, $\kappa$ is the constant. Micro-defects (imperfections) annihilate due to interaction with the ions of the substance according to the law of chemical kinetics

$$
\frac{\mathrm{d} N}{\mathrm{~d} \tau}=\nu N^{n} \exp \left(-\frac{E_{\nu}}{R T}\right),
$$

where $n$ is the order of the kinetics of imperfections annihilation, $E_{\nu}$ is the activation energy for removing imperfections, $\nu$ is the constant.

As we have shown in [14], the kinetics of the first order imperfections annihilation is realized for the sintering of ferrites. The data of other authors [15] confirm this conclusion. The model used in our analysis is based on the concept of isothermal sintering. However, we have shown earlier [14] that the theory also describes dynamic sintering with linear increase of the temperature. Subsequently this was confirmed in [16]. Therefore, we have hypothesized that heating mechanisms of sintering are the same as during the isothermal process, so the time of isothermal sintering in the basic formula may be substituted by the

$$
\tau=\tau_{\text {heat }}+\tau_{\text {is }},
$$

where $\tau_{\text {heat }}$ is the heating time, $\tau_{\text {is }}$ is time of isothermal soaking. The final expression describing the kinetics of sintering of ferrites is derived from the joint solution of Eqs. (2)-(3) (at $n=1$ ), by integration under the initial conditions, $v(\tau=0)=v_{\mathrm{pr}}$ is the pore volume in pressing and $N(\tau=0)=N_{0}$ is the concentration of imperfections in the beginning of sintering. The obtained result is

$$
\ln \frac{v_{\mathrm{pr}}}{v_{\mathrm{s}}}=\frac{\kappa N_{0}}{\nu} \exp \left(-\frac{E_{\kappa}-E_{\nu}}{R T}\right)
$$

$$
\times\left[1-\exp \left(-\left(\tau_{\text {heat }}+\tau_{\text {is }}\right) \nu\left(-\frac{E_{\nu}}{R T}\right)\right)\right] .
$$

Here $v_{\mathrm{pr}} / v_{\mathrm{s}}$ is the ratio of pore volume in pressing for the pore volume after sintering at the temperature $T$ during the isothermal soaking time $\tau_{\text {is }}$. Time of heating $\tau_{\text {heat }}$ and the parameters of kinetics $\kappa N_{0} / \nu, \nu$ as well as the activation energies of the processes $E_{\kappa}$ and $E_{\nu}$ are determined from the experiment.

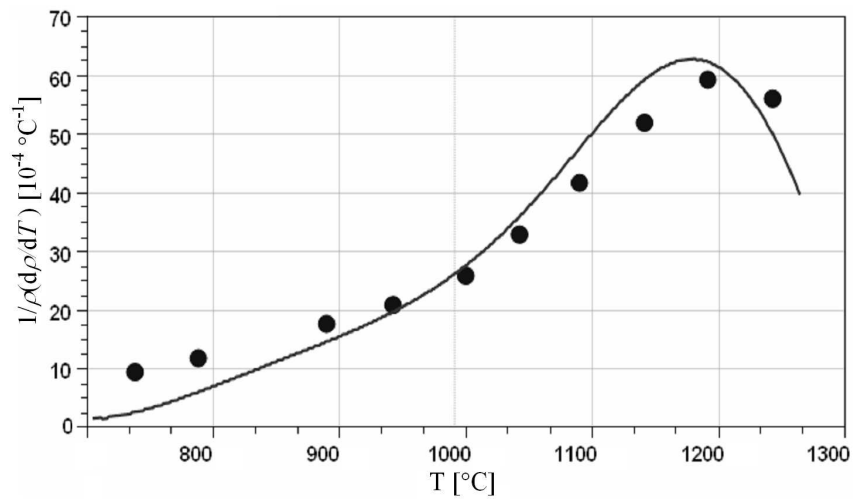

Fig. 1. Dependence of relative compacting zinc ferrite on the temperature during heating. Points are the experiment [17], line is a theory.

In order to confirm the validity of the theory at all stages of sintering we carried out an independent verification of expression (5) using experimental data obtained in [17]. As it is seen from Fig. 1, the theory satisfactorily describes the experimental data of sintering of zinc ferrite $\mathrm{ZnFe}_{2} \mathrm{O}_{4}$ on the stage of heating. At the stage of isothermal soaking formula (5) has been tested repeatedly by us [14] and other authors [18].

\section{Experiment description and data analysis}

Magnesium-zinc ferrites were produced by ceramic technology. Sintering of the ferrite samples were carried outdoors, that is in standard conditions with isothermal soaking at two temperatures: $1230^{\circ} \mathrm{C}$ and $1280^{\circ} \mathrm{C}$. Compactions were studied by the dilatometer. Heating was carried out in an accelerated mode, at which the thermal expansion was not taken into account [19]. The initial permeability was measured at frequency of $1 \mathrm{kHz}$ on the ferrite cores in the form of a ring with an outer diameter of $32 \mathrm{~mm}$.

Figure 2 shows the kinetic curves for the samples of the ferrite $\left(\mathrm{Mg}_{0.56} \mathrm{Zn}_{0.44}\right)_{1-y} \mathrm{Fe}_{2+2 y / 3} \mathrm{O}_{4}$ with $y=-0.05,0$, 0.05 . The calculated values of parameters of the kinetics are shown in Table.

As it is seen from the figure, the rate of compacting essentially depends on the stoichiometry of the sample. As it is evident from its formula, the stoichiometric MgZn-ferrite contains 3 metal cations on 4 anions. The number of anions counting on 3 cations in our ferrite is equal to $36 /(9-y)$. Consequently, when $y=-0.05$, the content 


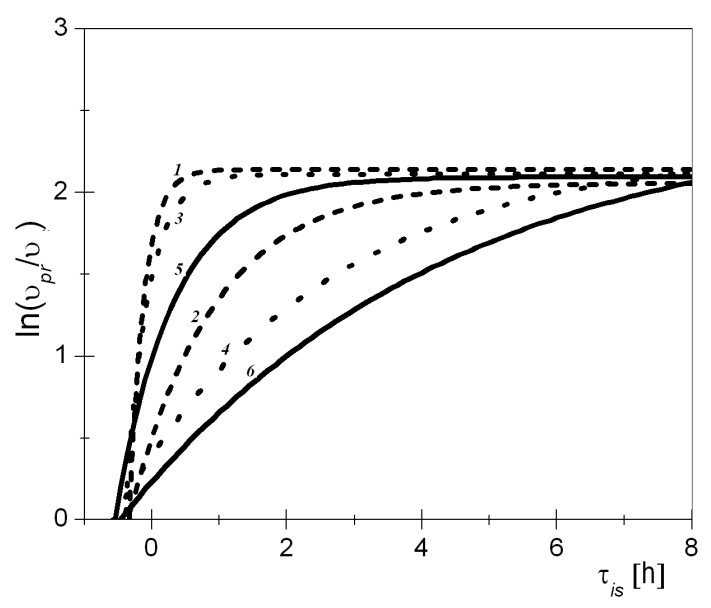

Fig. 2. Kinetics of compacting of ferrites $\left(\mathrm{Mg}_{0.56} \mathrm{Zn}_{0.44}\right)_{1-y} \mathrm{Fe}_{2+2 y / 3} \mathrm{O}_{4}$ during isothermal soaking. Calculated curves coincided with the experimental data within the thickness of lines. Here curves 2, 4, 6 are obtained at $1230{ }^{\circ} \mathrm{C}$, curves $1,3,5-$ at $1280^{\circ} \mathrm{C}$. Curves 1, 2 correspond to $y=-0.05$, curves 3,4 - to $y=0$ and curves 5,6 - to $y=0.05$.

TABLE

Parameters of kinetics of ferrites

$\left(\mathrm{Mg}_{0.56} \mathrm{Zn}_{0.44}\right)_{1-y} \mathrm{Fe}_{2+2 y / 3} \mathrm{O}_{4}$ sintering.

\begin{tabular}{c|c|c|c|c}
\hline \hline$y$ & $E_{\kappa}[\mathrm{kJ} / \mathrm{mol}]$ & $E_{\nu}[\mathrm{kJ} / \mathrm{mol}]$ & $\kappa N_{0} / \nu$ & $\tau_{\text {heat }}[\mathrm{h}]$ \\
\hline-0.05 & 594 & 662 & 6.84 & 0.3 \\
0 & 646 & 668 & 2.35 & 0.4 \\
0.05 & 695 & 678 & 0.0126 & 0.5
\end{tabular}

of anion vacancies corresponds to 0.022 . In this case the shrinkage rate is maximal (Fig. 2).

The model used here for analysis of the experimental data presumes that mass transfer is carried out due to the emergence and annihilation of so-called active imperfections $[13,20]$. They come from the genetic imperfections inherent for the powder. The relationship of generated active imperfections and annihilated genetic defects may not change during the time of sintering. Such a condition is described by the authors of [19] as quasi-equilibrium. In our case the ratio $\kappa N_{0} / \nu$ in principle can be considered as a quasi-equilibrium constant. Active imperfections are responsible for the pores decrease. Relative pores reduction rate $\left(-\frac{\mathrm{d} v}{\mathrm{~d} \tau} \frac{1}{v}\right)$, as follows from the Ivensen model, at a given temperature is proportional to the rate of active imperfections formation. According to (2) it is proportional to the parameter $\kappa N_{0}$. Relative rate of reduction of genetic defects, $\frac{\mathrm{d} N}{\mathrm{~d} \tau} \frac{1}{N}$, is proportional to $\nu$ in accordance with (3). It implies that the ratio of the given rates is equal to $\kappa N_{0} / \nu$.

Our results shown in Fig. 2 can be interpreted as follows. During sintering of ferrites with cationic vacancies $(y=0.05)$, the number of genetic defects (cation vacancies) is high and therefore the generation of the new active imperfections becomes weaker. This leads to decrease in the rate of sintering. Conversely, under the excess of anion vacancies, $y=-0.05$, the active imperfections such as cation vacancies are generated by intense sintering and activated. It increases the rate of compaction in the initial period of sintering (Fig. 2).

Let us note that the activation energy of both processes is reduced by substitution of iron ions for the ions of zinc and magnesium (Table). In our opinion it can be connected with higher diffusion mobility of $\mathrm{Zn}$ and $\mathrm{Mg}$ ions that is caused by more stable configuration of these ions in comparison with the iron ions configuration. It generally confirms the main conclusions of the Samsonov configuration theory.

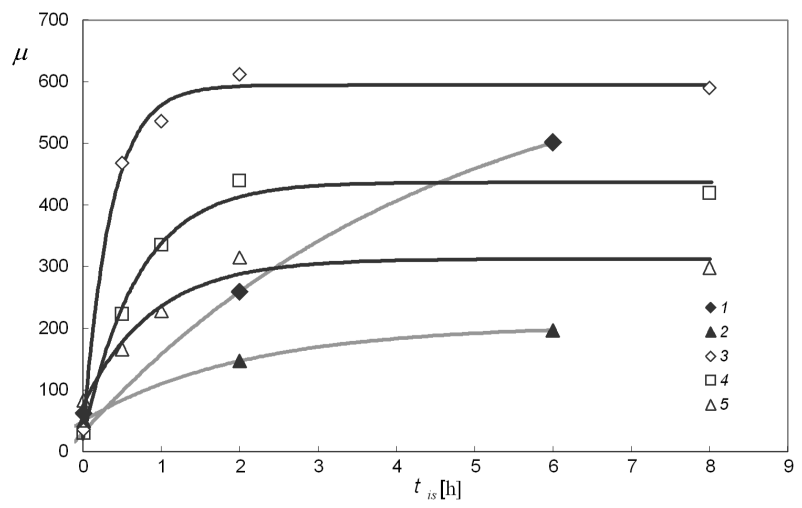

Fig. 3. Time dependence of the magnetic permeability of ferrites $\left(\mathrm{Mg}_{0.56} \mathrm{Zn}_{0.44}\right)_{1-y} \mathrm{Fe}_{2+2 y / 3} \mathrm{O}_{4}$ during isothermal soaking at the temperatures $1230{ }^{\circ} \mathrm{C}(1,2)$ and $1280^{\circ} \mathrm{C}(3,4,5)$. Curves 1,3 correspond to $y=-0.05$, curve 4 - to $y=0$ and curves 2,5 - to $y=0.05$.

The density of the samples and their magnetic permeability increase with the increase of the time of isothermal soaking (Fig. 3). The increase of permeability is mainly caused by decrease of the internal demagnetizing fields [8] in consequence of the reduction in the number of nonmagnetic pores in the magnetic ferrite. Thus, the process of sintering is an important step in the formation of the magnetic properties of ferrites.

\section{Conclusions}

The process of nonstoichiometric MgZn-ferrite sintering has been investigated. As it is shown, the rate of compaction increases with the substitution of iron cations with valence 3 on cations of magnesium and zinc with valence 2 . It is shown that the Ivensen model properly describes the experimental data and helps to reveal the mechanism of ferrites sintering. The process of ferrites sintering is described by using the Samsonov configuration theory. As it is shown, the magnetic permeability and the density of the studied chemical compositions increase with the increase of temperature and time of isothermal soaking. 


\section{References}

[1] E.M.M. Ibrahim, Appl. Phys. A 89, 203 (2007).

[2] Z. Pedsich, M.M. Bucko, M. Krolikowski, M. Bakalarska, J. Babiarz, J. Europ. Ceram. Soc. 24, 1053 (2004).

[3] J. Smit, H.P. Wijn, Adv. Electron. Electr. Phys. 6, 69 (1954).

[4] A. Gonchar, V. Andreev, L. Letuk, A. Shishkanov, V. Maiorov, J. Magn. Magn. Mater. 254-255, 544 (2003).

[5] G.V. Samsonov, in: Theory and Technology of Sintering, Ed. G.V. Samsonov, Naukova dumka, Kiev 1974, p. 10 .

[6] L.F. Pryadko, M.M. Ristič, Powder Metallurgy 1-2, 29 (2008).

[7] R. Morineau, M. Paulus, Phys. Status Solidi A 20, 373 (1973).

[8] A. Goldman, Modern Ferrite Technology, Springer Science + Business Media, Inc., Pittsburg 2006.

[9] L.A. Bashkirov, V.V. Pankov, Mechanism and Kinetics of Formation of Ferrits, Nauka i Technika, Minsk 1988.

[10] A.A. Sattar, A.H. Wafik, K.M. Kandil, J. Phys. D, Appl. Phys. 29, 25 (1996).
[11] V.Ya. Gegusin, Sintering Physics, Nauka, Moscow 1967.

[12] V.A. Ivensen, J. Techn. Phys. 40, 36 (1970).

[13] V.A. Ivensen, Powder Metallurgy 8, 47 (1993).

[14] A.V. Kopayev, B.I. Kindrat, L.M. Letyuk, in: Proc. Workshop on Powder Metallurgy, PPI, Penza (Russia) 1990, p. 68.

[15] Yu.D. Tretyakov, Solid-Phase Reactions, Chemistry, Moscow 1978.

[16] P. Roura, J. Costa, J. Farjas, Mater. Sci. Eng. A 337, 248 (2002).

[17] M. Rahman, L.C. De Junghe, J. Am. Ceram. Soc. 76, 1739 (1993).

[18] E.V. Markovsky, E.M. Kriger, L.A. Bashkirov, A.I. Rudskoy, L.Z. Melamed, in: Obtaining $\mathrm{Ma}$ terials for Electronic Engineering and Their Study, NIITECIM, Moscow 1982, p. 105.

[19] K. Maca, V. Pouchly, A.R. Boccaccini, Science of Sintering 40, 117 (2008).

[20] V.Z. Belen'kii, Powder Metallurgy 8, 56 (1993). 